\title{
Quality Of Life of Hypertensive Patients on Different Types of Antihypertensive Medications
}

\author{
Sawsan Shanableh ${ }^{1}$, Abdulmula Abdulkarem ${ }^{2}$, Mohammed Shamssain ${ }^{3}$, \\ Fadi Sarhan ${ }^{4}$ \\ ${ }^{1}$ Lecturer, Clinical Pharmacy and Pharmacy Practice Department, Pharmacy College, Ajman University of \\ Science and Technology Network, Ajman, United Arab Emirates. \\ 2 Associate Professor, Pharmacy Practice and Pharmacotherapeutics Department, Pharmacy College, \\ University of Sharjah, Sharjah, United Arab Emirates. \\ 3 Associate Professor, Clinical Pharmacy and Pharmacy Practice Department, Pharmacy College, Ajman \\ University of Science and Technology Network, Ajman, United Arab Emirates. \\ 4 Healthcare Dealer/Market Manager-Middle East \& Africa, Hermanmiller Healthcare company, Duabai, \\ United Arab Emirates.
}

\begin{abstract}
More than three quarter of the total death in the UAE is due to cardiovascular diseases. Health Related Quality of Life (HRQL) is a broad multidimensional concept that usually includes subjective evaluations of both positive and negative aspects of life. The aim of the study was to evaluate the quality of life for hypertensive patients using different types of antihypertensive medications. A cross-sectional, randomized study was conducted in Ajman and Sharjah, October 2012 to May 2013, using a self-completed questionnaire and SF-36 HRQL questionnaire by 150 hypertensive patients on different types of medications and 220 control subjects. Around 53.3\% of hypertensive patients used a combination therapy of two medicines; $33 \%$ used Angiotensin receptor blockers $(A R B)+$ Diuretics and $20 \%$ used the combination of B-blockers + Diuretics. Only $6 \%$ of the patients used B-Blockers class for their treatment. Data were analyzed by SPSS. The mean values of Physical Functioning (PF), Role-Physical (RP), Bodily Pain (BP), General Health (GH), Vitality (V), Social Functioning (SF), Role Emotional (RE), Mental Health (MH), Physical Components Summary (PCS), and Mental Components Summary (MCS) scores were 62.5, 62.9, 72.1, 62.1, 64.5, 76.7, 68.8, 66.2, 47.0, and 47.8, respectively. Patients who were using a combination therapy of Angiotensin receptors blockers (ARB) plus Diuretics had the highest score in all SF-36 scales compared to other medications. Patients who were using Calcium channel blockers (CCB) had the lowest self-reported health compared to other medications. There were no significant differences between using single therapy of Angiotensin converting enzyme inhibitors (ACEI) or Angiotensin receptors blockers (ARB) and using a combination therapy of B-blockers + Diuretics; they almost had the same scores on most of the scales. Less than half (35.3\%) of medication users suffered side effects from their medications and the common side effects they experienced were nausea (1.3\%) and cough (34\%). Early recognition of participants with this chronic disease is important in clinical disease management and in participation in education and intervention programs aimed at improving their HRQL.
\end{abstract}

KEYWORDS - Health related quality of life (HRQL), Hypertension, Antihypertensive medications, SF-36 questionnaire, control subjects.

\section{INTRODUCTION}

Cardiovascular diseases (CVD) are the leading cause of death and disability across the globe in developed and developing countries. It's affecting men and women for almost the same extent as an estimated 17.3 million people died from CVDs in 2008, representing 30\% of all global deaths [1]. The prevalence of these diseases is raising most quickly in Southeast Asia and the Eastern Mediterranean regions [1,2]. In UAE, more than three quarters of the total death was due to CVDs. This is because of high incidence of risk factors such as diabetes (25\%), hypertension (30\%), overweight and obesity (50\%), high cholesterol (30 to 40\%), poor diet (e.g. low intake of fruit and vegetables) (31\%), physical inactivity (22\%), and bad smoking habit $(22 \%)[2,3]$.

Health Related Quality of Life (HRQL) is a broad multidimensional concept that usually includes subjective evaluations of both positive and negative aspects of life [4]. The concept of HRQL and its determinants have developed since the 1980s to include those aspects of overall quality of life that can be clearly shown to affect health - either physical or mental $[5,6]$. There are many instruments used for evaluating the HRQL, example of these instruments are the Medical Outcomes Study Short Forms: SF-12 and SF-36 [4]. 
Measuring HRQL can help determine the burden of preventable disease, injuries, and disabilities, and it can provide valuable new insights into the relationships between HRQL and risk factors, such as self-reported chronic diseases (diabetes, breast cancer, arthritis, and hypertension), and their risk factors (body mass index, physical inactivity, and smoking status) [5].

Hypertension has detrimental effects on a range of health outcomes including HRQL [7, 8]. There have been number of studies examining the impact of cardiovascular diseases either single or co-morbid conditions on the HRQL, especially the co-morbidity of hypertension [9, 10]. However, none of these studies aimed to elicit this problem in people with hypertension in the UAE. The aims and subjects of the present study are to compare the HRQL between hypertensive and healthy subjects in the UAE using the SF-36 short form questionnaire and investigate which class of Antihypertensive medications improves patients' quality of life more than other classes.

\section{MATERIALS AND METHODS}

A descriptive cross-sectional, randomized study was conducted on the public population of Northen Emirates (Ajman and Sharjah) in UAE over a period of eight months (October 2012 to May 2013) by means of interviews carried out by trained pharmacists with proper skills for the study and distributing the questionnaires and recollecting them after self-completing by the participants. The study was approved by Ajman University Ethical Committee. The permission to use the Health quality survey form of SF-36 v2 and its scoring system was requested and obtained from QualityMetric Inc. center in US. (License agreement\#QM018670 \& QM019018). All subjects were asked for their consent in order to participate in the study.

A total of 150 hypertensive patients and 220 healthy subjects were randomly selected. Inclusion criteria were healthy subjects and patients with age of 35 years old or older, male or female and the patients were diagnosed with hypertension only. The exclusion criteria were all subjects who were less than 35 years old, patients with co morbidity conditions other than hypertension, pregnant women and individuals with severe acute or chronic mental disorders. The response rate was $75 \%$.

The SF-36 v2 Health Survey Questionnaire was used for evaluating the Health Related Quality of Life (HRQL) in healthy and non-healthy general population. SF-36 is a fixed-format self-completed questionnaire that contains 36 items integrated in multi-item scales measuring eight general health scales: physical functioning (PF); role-physical (RP); bodily pain (BP); general health (GH); vitality (VT); social functioning $(\mathrm{SF})$; role-emotional (RE) and mental health $(\mathrm{MH})$. These eight scales may be further aggregated into two summary measures: the physical component summary measure (PCS) and the mental component summary measure (MCS), which are used to compare the HRQL between control and patients group.

The questionnaire included two parts. The first part was composed of 6 questions which covered the Socio-demographic characteristics: age, gender, marital status, disease state, medications used along with their side effects. The second part was related to general physical and emotional health and it included 36 questions used to evaluate the quality of life in healthy subjects and hypertensive patients population that participated in the study.

DATA ANALYSIS: All Socio-demographic data was analyzed using the Statistical Package for the Social Sciences (SPSS, version 20 for Windows) computer program. Descriptive analysis included calculations of means, $95 \%$ confidential intervals (CI) of means and frequencies of categorical variables. Non-parametric tests and comparative means tests (T-test; ANOVA) were used for the analysis and evaluation. The SF-36 v2 questions were analyzed by the QualityMetric Health Outcomes TM Scoring software 4.5. The software uses all the 36 items to produce scores for the PCS-36 and the MCS-36 scales and applies a norm-based scoring algorithm empirically derived from the data of a US general population survey. In theory, the subscale scores of SF-36 range from 0 to 100, with higher scores signifying greater perceived health and the lower one reflecting lower health; 0: the worst and 100: the best. P-value $\leq 0.001$ was considered statistically significant. To test reliability, the internal consistency for the eight scales domains and for summary measures were estimated using Cronbach's alpha coefficient and alpha equal to or greater than 0.70 was considered satisfactory. The Cronbach's alpha exceeded 0.9 for all scales, which indicates high internal consistency.

\subsection{Socio-demographic and clinical characteristics:}

\section{RESULTS}

Only respondents with complete data sets were included in this study. Participants' Sociodemographic and clinical characteristics are presented in Table 1. The range of age of participants was 41-50 years; 55\% control group and 34.7\% patients group. Majority of the participants, were female in both control and patients groups, $64.5 \%$ and $56 \%$, respectively. Most of the participants were married, $89.1 \%$ and $97.3 \%$ in control and patients groups, respectively. Most of healthy subjects didn't use any medications. Almost all patients used medications for their disease control and only $1.3 \%$ of them didn't take any medicine. 
Table 1: Socio-demographic data of the respondents

\begin{tabular}{|l|l|l|}
\hline Variables & \multicolumn{1}{|c|}{$\begin{array}{c}\text { Control Group (220) } \\
\text { N (\%) }\end{array}$} & \multicolumn{1}{|c|}{$\begin{array}{c}\text { Hypertensive Patients (150) } \\
\text { N (\%) }\end{array}$} \\
\hline Age & \multicolumn{1}{|c|}{} & $11(7.3)$ \\
$35-40$ & $56(25.5)$ & $52(34.7)$ \\
$41-50$ & $121(55)$ & $87(58)$ \\
$\geq 51$ & $43(19.5)$ & $66(44)$ \\
\hline Gender & & $84(56)$ \\
Male & $78(35.5)$ & $2(1.3)$ \\
Female & $142(64.5)$ & $2(1.3)$ \\
Marital status & $12(5.5)$ & $146(97.3)$ \\
Single & $5(2.3)$ & 0 \\
Engaged & $196(89.1)$ & $148(98.7)$ \\
Married & $7(3.2)$ & $2(1.3)$ \\
Divorced & $10(4.5)$ & \\
\hline Usage of any medications & $210(95.5)$ & \\
Yes & & \\
No & & \\
\hline
\end{tabular}

Tables 2 summarized the types of medications used by patients to control hypertension. Antihypertensive medications prescribed were mainly combination therapy in $53.3 \%$ of respondents. Thirty three percent of them used Angiotensin receptor blockers (ARB) + Diuretics and 20\% used the combination of Bblockers + Diuretics. Only 6\% of the patients used B-Blockers for their treatment. Less than half (35.3\%) of medication users suffered side effects from their medications and $64.7 \%$ had no side effects reported. The common side effects they experienced were nausea (1.3\%) and cough (34\%).

Table 2: Types of medications used by Hypertensive patients and any side effects they suffered

\begin{tabular}{|l|c|c|}
\hline Types of medications used & $\mathbf{N}$ & \% \\
\hline No medications & 2 & 1.3 \\
\hline ACEI & 19 & 12.7 \\
\hline CCB & 16 & 6.7 \\
\hline B-Blockers & 9 & 16 \\
\hline ARB & 24 & 33.3 \\
\hline ARB + Diuretics & 50 & 20 \\
\hline B-Blockers + Diuretics & 30 & 35.3 \\
\hline Side effects & \multicolumn{2}{|}{} \\
\hline Yes & 53 & 64.7 \\
\hline No & 97 & 64.7 \\
\hline Types of side effects & 97 & 1.3 \\
\hline No any side effects & 2 & 34 \\
\hline Nausea & 51 & \\
\hline Cough & \multicolumn{1}{|}{} \\
\hline $\begin{array}{l}\text { ACEI: Angiotensin converting enzyme inhibitors, CCB: calcium channels blocker, } \\
\text { B-Blocers: Beta-Blockers, ARB: angiotensin receptors blockers. }\end{array}$ \\
\hline \multicolumn{2}{|c|}{} \\
\hline \multicolumn{1}{|c|}{} \\
\hline
\end{tabular}

\subsection{Health Related Quality of Life Outcomes}

\subsubsection{SF-36 profiles by total population}

Table 3 displays the mean ( \pm S.D) of the Medical Outcomes Study Short Form 36 questionnaire and the physical and mental components summary scores among control group and disease group. Each of the eight subscales and physical and mental components summary scales were scored on a scale from $0-100$, zero being the lowest and poorest score and 100 the highest and best possible score. The highest scores were reported for control group in all scales measured.

ANOVA test was performed and the results showed that there were a very high significance differences $(p \leq 0.001)$ in the eight scales of quality of life and in physical and mental components summary scores among control group and disease group; control group had higher scores than hypertensive patients. 
Table 3: Mean ( \pm S.D) of respondents' Quality of Life (QOL) domain score

\begin{tabular}{|l|l|l|}
\hline Scale / Number of sample & \multicolumn{1}{|c|}{ Control Participants (220) } & \multicolumn{1}{|c|}{ Hypertensive Patients (150) } \\
\hline Physical Functioning & $96.6817(3.95680)$ & $62.5317(23.64813)$ \\
\hline Role-Physical & $96.7898(8.66519)$ & $62.9583(32.31894)$ \\
\hline Bodily Pain & $91.4682(12.04307)$ & $72.0600(12.64817)$ \\
\hline General Health & $80.7909(11.55103)$ & $62.1200(15.75224)$ \\
\hline Vitality & $83.1534(11.21057)$ & $64.5000(13.57230)$ \\
\hline Social Functioning & $90.2841(13.08795)$ & $76.7500(20.01625)$ \\
\hline Role Emotional & $97.5760(9.03410)$ & $68.8899(32.82260)$ \\
\hline Mental Health & $85.5000(8.69183)$ & $66.2667(19.92593)$ \\
\hline Physical Components Summary & $57.2626(2.39184)$ & $47.0584(6.40690)$ \\
\hline Mental Components Summary & $56.0771(4.08052)$ & $47.8135(9.98601)$ \\
\hline
\end{tabular}

\subsubsection{SF-36 profiles by Types of medications used in Hypertensive patients}

The effects of different types of medications taken for each disease state may deteriorate the HRQL for patients more than the disease itself or it can improve the health state of patients.

Figure 1 shows that hypertensive patients who were using a combination therapy of Angiotensin receptors blockers (ARB) + Diuretics had the highest score in all SF-36 scales compared to other medications. Patients who were using Calcium channel blockers (CCB) had the lowest self-reported health compared to other medications. There were no significant differences between using single therapies of Angiotensin converting enzyme inhibitors (ACEI) or Angiotensin receptors blockers (ARB) and using a combination therapy of Bblockers + Diuretics; they almost had the same scores on most of the scales.

Figure 1: SF-36 profiles by Types of medications used in Hypertensive patients:

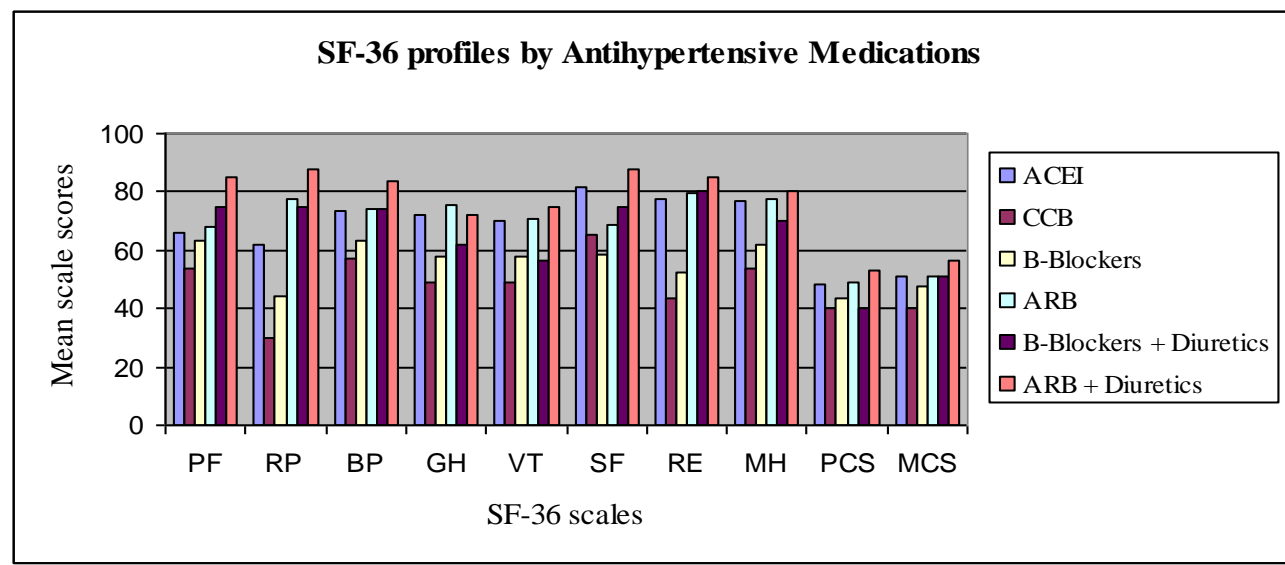

\section{DISCUSSION}

In view of vagueness about the pathophysiology of many cardiovascular disorders, their association with lifestyle in different societies is of a particular interest. The rapid lifestyle changes that have taken place in the Gulf Region offer a unique opportunity to study the effect of such changes on CVDs morbidity patterns and risk factors that affect the quality of health of individuals.

This study shows that in the UAE, despite a modern and accessible health infrastructure, the level of hypertension is high which is similar to the situation found in many other places in the world, both in developed and developing countries. It shows that participants with hypertension reported comparably limited HRQL in all dimensions of SF-36, compared with healthy individuals and this explains that this condition impose negative limitations in physical and mental functioning as found in the study carried out by Poljičanin, Tamara et al [10]. However, available literature [11] showed that hypertension had a little association with mobility and physical activities and it is more strongly related to anxiety and depression, while our study found that patients with hypertension had lower scores in all scales of SF-36, even the physical health. This might suggest that assessing HRQL may be beneficial even in the early stages of treatment for hypertension. 
Treatment of the disease state of the patient by choosing the suitable type of medications is a key strategy for the prevention of cardiovascular disease. In this study the HRQL scores were different among different types of medications used for hypertension treatment. Significant differences in HRQL scores between treatments were observed in our study. Recent data $[12,13]$ suggest that patients with established hypertension receiving pharmacotherapy may feel better as blood pressure is lowered because of symptoms improvement, regardless of the type of the medication given. The current study's results are consistent with previous studies evaluating the effect of antihypertensive medications on HRQL. Our study suggests that using a combination therapy of Angiotensin receptors blockers (ARB) + Diuretics had the highest QOL and the patients who were using Calcium channel blockers (CCB) had the lowest QOL. Also, it showed that using Angiotensin converting enzyme inhibitors (ACEI) or Angiotensin receptors blockers (ARB) and using a combination therapy of Bblockers + Diuretics had high scores on all scales of SF-36. These results are similar to results found by other studies, [14, 15] which found that Angiotensin-converting enzyme inhibitors and angiotensin II receptor antagonists may offer some advantage with regard to effects on cognitive function and sexual activity over other classes of medications while calcium channel blocker-based treatment regimen lowered HRQL more than other types of medications. Also, $[12,16]$ showed that patient symptoms and HRQL improve with control of blood pressure measures with diuretics and other medications. Adverse effects are commonly a cause of therapy discontinuation in many conditions including hypertension. A treatment regimen that maintains patients' HRQL is more likely to be taken regularly, with potential additional benefits. Pharmacological interventions in patients with established hypertension with many medications, including ARBs, suggest a great impact on HRQL in treated patients compared with placebo [16].

\section{CONCLUSION}

CONCLUSION: Hypertension is an important preventable cause of death and the treatment of this disease is a key strategy for the prevention of cardiovascular diseases. Descriptions of patients' HRQL among different diagnosis groups could be used by clinicians to assist individualized patient care. Our results showed an adverse impact of hypertension on participants' well-being and functioning. This could be useful in clinical practice, particularly in early diagnosis and treatment of hypertension, at which point improving self-management and consequently HRQL is still possible. Also it can be useful in the prevention of complications associated with hypertension such as: cerebrovascular disease and chronic kidney disease, which lead to further reduction in HRQL.

RECOMMENDATIONS: based on the present study, the HRQL of patients having hypertension can be improved by healthy life style and using suitable medications. There are several recommendations that can be offered to improve the HRQL among these patients; increase awareness on health promotion and hypertension among population, especially those people with low income and education levels, regular checkup, which can help in early diagnosis of any health problem and maintaining the HRQL of the individual in high level, following the guidelines in the priority of choosing the medications used in treatment of patients with hypertension. Also, increasing the sample size in patient group to get more clear information about each class of medications used and their effects on quality of life of patients.

LIMITATIONS: There are several limitations that may have influenced the results of this study. The SF-36 form is a questionnaire designed for self-administration, but in many cases in our study, the forms were administered to the subjects by an interview, which could have affected the results. It has been shown that interviewers (e.g. relatives, friends, etc) tend to underestimate the condition of subjects. Also, because of the self-reported of sf-36 data, more detailed information on disease duration, adherence to treatment and other complications were not available. As these are generic instruments, they may not be as responsive to a change in treatment as other more specific instruments [17]. Another factor that could have affected the results of this study was the mood state of subjects at the time of the interview. The psychological state of the subjects like anxiety and depression could have affected the data provided by them especially the respondents who were staying in the hospital recently.

SUGGESTIONS FOR FURTHER REASEARCHES: Further research on appropriate interventions aimed at HRQL of these participants is needed. Also, additional future works are needed to increase adherence to lifestyle counseling for all hypertensive patients.

\section{ACKNOWLEDGEMENTS}

We are grateful to the patients who had spent their valuable time in participating in this study and to pharmacists who help us in distributing the questionnaires to all participants. 


\section{REFRENCES}

[1] World Health Organization. Cardiovascular diseases (CVDs) fact sheet $N^{\circ} 317$, September 2012. Available at this website: http://www.who.int/mediacentre/factsheets/fs317/en/index.html (Updated September, 2009 and accessed on 5/11/2012).

[2] Halpin, H. A., Morales-Suarez-Varela, M. M., \& Martin-Moreno, J. M. Chronic disease prevention and the new public health. Public Health Reviews, 32(1), 2010, 120-154.

[3] World Health Organization. The world health report 2002. Reducing risks, promoting healthy life. Geneva: World Health Organization, 2002.

[4] Centra, L. New information about health-related quality of life. NACCHO News, 1998

[5] Centers for Disease Control and Prevention. Measuring healthy days: Population assessment of health-related quality of life. Centers for Disease Control and Prevention, Atlanta, Georgia 2000.

[6] Selim, A. J., Rogers, W., Fleishman, J. A., Qian, S. X., Fincke, B. G., Rothendler, J. A., \& Kazis, L. E. Updated US population standard for the Veterans RAND 12-item Health Survey (VR-12). Quality of Life Research, 18(1), 2009, 43-52.

[7] Harris, M. I. Health care and health status and outcomes for patients with type 2 diabetes. Diabetes Care, 23(6), 2000, 754-758.

[8] Reddy, S. S. Health outcomes in type 2 diabetes. International journal of clinical practice. Supplement, (113), $2000,46-53$.

[9] Arredondo, Shelley A., et al. Impact of comorbidity on health-related quality of life in men undergoing radical prostatectomy: Data from CaPSURE. Urology, 67(3), 2006, 559-565.

[10] Poljičanin, T., Ajduković, D., Šekerija, M., Pibernik-Okanović, M., Metelko, Ž., \& Mavrinac, G. V. Diabetes mellitus and hypertension have comparable adverse effects on health-related quality of life. BMC Public Health, 10(1), $2010,12$.

[11] Theodorou, M., Kaitelidou, D., Galanis, P., Middleton, N., Theodorou, P., Stafylas, P., ... \& Maniadakis, N. Quality of life measurement in patients with hypertension in cyprus. The Hellenic Journal of Cardiology, 52(5), 2011, 407-415.

[12] Erickson, S. R., Williams, B. C., \& Gruppen, L. D. Perceived symptoms and health-related quality of life reported by uncomplicated hypertensive patients compared to normal controls. Journal of human hypertension, 15(8), 2001, 539-548.

[13] Dahlöf, B., Degl'Innocenti, A., Elmfeldt, D., Puig, J. G., Gundersen, T., Hosie, J., ... \& Wiklund, I. Felodipine--Metoprolol Combination Tablet: Maintained Health-Related Quality of Life in the Presence of Substantial Blood Pressure Reduction\&ast. American journal of hypertension, 18(10), 2005, 1313-1319.

[14] Fogari, R., \& Zoppi, A. Effect of antihypertensive agents on quality of life in the elderly. Drugs \& aging, 21(6), 2004, 377-393.

[15] Williams, S. A., Michelson, E. L., Cain, V. A., Yang, M., Nesbitt, S. D., Egan, B. M., \& Julius, S. An Evaluation of the Effects of an Angiotensin Receptor Blocker on Health-Related Quality of Life in Patients With High-Normal Blood Pressure (Prehypertension) in the Trial of Preventing Hypertension (TROPHY). The journal of Clinical Hypertension, 10(6), $2008,436-442$.

[16] Neaton, J. D., Grimm, R. H., Prineas, R. J., Stamler, J., Grandits, G. A., Elmer, P. J., ... \& Monske, M. Treatment of mild hypertension studyfinal results. JAMA: the journal of the American Medical Association, 270(6), 1993, 713-724.

[17] Bulpitt, C. J., \& Fletcher, A. E. The measurement of quality of life in hypertensive patients: a practical approach. British journal of clinical pharmacology, 30(3), 1990, 353-364. 